\title{
Parasites modulate within-colony activity and accelerate the temporal polyethism schedule of a social insect, the honey bee
}

\author{
Myrsini E. Natsopoulou ${ }^{1} \cdot$ Dino P. McMahon ${ }^{2,3} \cdot$ Robert J. Paxton $^{1}$
}

Received: 16 August 2015 / Revised: 24 September 2015 / Accepted: 29 September 2015 / Published online: 7 October 2015

(C) Springer-Verlag Berlin Heidelberg 2015

\begin{abstract}
Task allocation in social insect colonies is generally organised into an age-related division of labour, termed the temporal polyethism schedule, which may in part have evolved to reduce infection of the colony's brood by pests and pathogens. The temporal polyethism schedule is sensitive to colony perturbations that may lead to adaptive changes in task allocation, maintaining colony homeostasis. Though social insects can be infected by a range of parasites, little is known of how these parasites impact within-colony behaviour and the temporal polyethism schedule. We use honey bees (Apis mellifera) experimentally infected by two of their emerging pathogens, Deformed wing virus (DWV), which is relatively understudied concerning its behavioural impact on its host, and the exotic microsporidian Nosema ceranae. We examined parasite effects on host temporal polyethism and patterns of activity within the colony. We found that pathogens accelerated the temporal polyethism schedule, but without reducing host behavioural repertoire. Infected hosts exhibited increased hyperactivity, allocating more time to self-
\end{abstract}

Communicated by D. Naug

Electronic supplementary material The online version of this article (doi:10.1007/s00265-015-2019-5) contains supplementary material, which is available to authorized users.

Myrsini E. Natsopoulou

myrsini.natsopoulou@zoologie.uni-halle.de

1 Institut für Biologie, Martin-Luther-Universität Halle-Wittenberg, Hoher Weg 8, 06120 Halle (Saale), Germany

2 Institute of Biology, Free University Berlin, Schwendenerstr.1, 14195 Berlin, Germany

3 Department for Materials and Environment, BAM Federal Institute for Materials Research and Testing, Unter den Eichen 87, 12205 Berlin, Germany grooming and foraging-related tasks. The strength of behavioural alterations we observed was found to be pathogen specific; behavioural modifications were more pronounced in virus-treated hosts versus $N$. ceranae-treated hosts, with potential benefits for the colony in terms of reducing withincolony transmission. Investigating the effects of multiple pathogens on behavioural patterns of social insects could play a crucial role in understanding pathogen spread within a colony and their effects on colony social organisation.

Keywords Host · Pathogen · Multiple infection · Apis mellifera $\cdot$ Nosema ceranae $\cdot$ Deformed wing virus

\section{Introduction}

Animal species are targets of a wide range of parasite species that can have a profound impact on the physiology and behaviour of the infected individual (Hart 1990; Moore 2002). Host behaviour alterations mediated by the parasite can either be a fitness-neutral side effect of infection or involve manipulation that enhances parasite transmission (Poulin 2006). They can be also mediated by the host to minimise fitness costs or to protect conspecifics (Hart 1990; Poulin 2006). In either case, altered host behaviour (e.g. reduced mobility, change of habitat preference) can have direct effects on the host's population structure and community function (Mouritsen and Poulin 2002; Mouritsen and Poulin 2010). The effects of pathogens on the behaviour of social insects such as honey bees (Apis mellifera) are of particular interest as social insects live at high densities in colonies of highly related individuals, which may facilitate pathogen transmission and impact. Yet they have also evolved group-level defences against pathogens known as 'social immunity' (Cremer et al. 2007) that may help them reduce the impact of 
pathogens. Many of these social immunity defences are behavioural and can have both prophylactic and post-infection roles in limiting the spread of a pathogen (Cremer et al. 2007; Evans and Spivak 2010).

Honey bee workers, like many eusocial insects, exhibit age-related biases in task performance, known as temporal polyethism, with young individuals performing tasks inside the colony and older workers specialising in external duties such as foraging and guarding (Winston 1987). There are four well-recognised and described temporal castes: the cell cleaners (generally from 1 to 4 days of age), the nurses (412 days old) where workers take care of the brood, the middle aged bees (12-21 days old) that perform tasks related to nest maintenance and food storing and finally the foragers (Seeley 1982; Johnson 2008). Ageing and the transition from one temporal caste and set of tasks to another is gradual and it is partially correlated with physiological alterations such as changes in juvenile hormone and vitellogenin titres (Robinson 1987; Huang and Robinson 1992; Schulz et al. 2002).

Several mechanisms have been proposed that may trigger temporal shifts in task (reviewed by Gordon 1996; Beshers and Fewell 2001; Johnson 2010); information flow through behavioural interactions among individuals seems to be an important component of coordination within a hive. However, there is great flexibility as bees can undertake tasks from several temporal castes in quick succession, skip castes or revert from foraging to nurse tasks depending on the needs of the colony (e.g. food availability) (Huang and Robinson 1992; Wegener et al. 2009; Johnson and Frost 2012). The system is sensitive to perturbations and several stressors can trigger a change in the usual progression such as food availability and colony demography (Oster and Wilson 1978; Robinson 1992; Huang and Robinson 1996).

Longevity has been found to be associated with the speed that a honey bee progresses through its temporal polyethism schedule (Woyciechowski and Kozłowski 1998; Tofilski 2009). Thus, individuals with reduced lifespan due to e.g. injury or infection have been found to adjust their entire schedule, with the consequence that they perform the risky task of foraging (i.e. the last task in the temporal polyethism schedule) earlier in life compared to healthy individuals (Woyciechowski and Kozłowski 1998; Woyciechowski and Moron 2009). However, it should be noted that reduced longevity could also be a side effect of an earlier behavioural switch to precocious foraging caused by a stressor (Rueppell et al. 2007). Thus, when disease causes a speeding up of the temporal polyethism schedule, this can have adaptive benefits for the colony by accelerating an infected worker into precocious foraging (Tofilski 2009) so that it is less likely to infect sensitive brood with pathogens (the conveyor belt model of Schmid-Hempel (1998)).

Honey bees are susceptible to a wide range of pathogens that can affect both their physiology and their expected longevity (Chen and Siede 2007; Evans and Schwarz 2011). Workers infected with Nosema spp. engage in outside-nest duties, such as foraging or guarding, earlier than their noninfected nestmates (Woyciechowski and Kozłowski 1998; Goblirsch et al. 2013), possibly due to energetic stress (Mayack and Naug 2009; Mayack and Naug 2011) or other physiological pathways (Goblirsch et al. 2013). The same trend has been observed in bees infected by other common honey bee pathogens such as Varroa destructor or sacbrood virus (Bailey and Fernando 1972; Downey et al. 2000). However, the opposite pattern has also been observed, with one pathogen, the tracheal mite Acarapis woodi, slowing down the temporal polyethism schedule by lowering the metabolic rate of infected individuals (Downey et al. 2000). Hence, a general rule in predicting how pathogens alter temporal polyethism is difficult to make.

In our study, we focus on two common and emergent honey bee pathogens, the microsporidian Nosema ceranae and Deformed wing virus (DWV). Both pathogens are widespread in honey bees, and their distribution and prevalence has increased during the last decades (Klee et al. 2007; Carreck et al. 2010; Genersch and Aubert 2010; Martin et al. 2012). In addition, both pathogens have been associated with reduced lifespan of adult workers (Higes et al. 2007; Dainat et al. 2012; Natsopoulou et al. 2015). N. ceranae is an infection of adult bees only and is transmitted between adults per os as bees ingest spore-laden faeces (Fries 2010). DWV infects all stages (larva, pupa, adult), and though nowadays primarily transmitted by the intermediary vector $V$. destructor from adult to adult or pupa, it can also be passed from adult to adult via trophallaxis and from adult to larva during larval feeding (de Miranda and Genersch 2010). Previous studies on the effects of Nosema spp. on temporal polyethism have mainly focused on the transition between inside to outside-nest duties, and precocious foraging has been well documented (Woyciechowski and Moroń 2009; Goblirsch et al. 2013). Effects on within-hive tasks have only recently received attention (McDonnell et al. 2013; Retschnig et al. 2015), but no significant alterations in social behaviours have been observed. Considerably less information is available on the behavioural effects of DWV on honey bees. The virus has been found to be present and to replicate in several locations of the honey bee brain (Shah et al. 2009), and a link between DWV infection and aggression or learning deficits has been proposed (Iqbal and Mueller 2007; but see Rortais et al. 2006). Moreover, there is evidence that DWV-infected individuals can be actively recognised and removed from the colony by their healthy nestmates, at least when they display obvious symptoms (e.g. severe wing deformities) of infection (Baracchi et al. 2012).

As co-infections of $N$. ceranae and DWV are common in nature, both at the colony and the individual bee level (CoxFoster et al. 2007; Fürst et al. 2014), our aim was to investigate 
the effect of these two pathogens, alone or as a mixed infection, on the honey bee temporal polyethism schedule, social behaviours and within-colony activity patterns. To our knowledge, our study is the first to address the impact of DWV infection, in addition to the effect of pathogen co-infection, on within-hive honey bee behaviour. We used scan sampling to document the behaviour of individually tagged workers and determined if experimental infection treatments triggered a change in the temporal polyethism schedule, either by altering its speed or by causing infected individuals to skip duties. Finally, we also tested whether there are quantitative differences in patterns of behaviour across treatments, which could give an insight to the contribution of infected individuals to colony performance.

\section{Material and methods}

\section{Setup of colonies}

Four unrelated queenright colonies of $A$. mellifera, as used by local beekeepers (subspecies carnica), were placed in threeframe observation hives 1 month prior to the beginning of the experiment. Observation hives were kept in a room, with bees allowed access to the outdoors via holes in the walls. All four colonies had the same layout. The two bottom frames comprised the brood area, while the top frame contained a mix of open and capped honey cells. A differently coloured/patterned card was attached below nest entrances to increase entrance recognition and reduce drifting (Free and Spencer-Booth 1961).

\section{Experimental setup}

Honey bee brood was obtained from three additional, unrelated colonies and emerged overnight at $34^{\circ} \mathrm{C}$ in the laboratory. One-day-old bees were subjected to injections between the third and fourth abdominal segment with $1 \mu$ l containing either $10^{7}$ particles of DWV in $0.5 \mathrm{M}$ potassium phosphate buffer pH 8.0 (PBS) or a control solution. DWV inoculum originated from symptomatic bees found in our apiary and was propagated into uninfected pupae as described in Doublet et al. (2015b). Control inoculum was prepared following the same protocol using uninfected pupae. Inocula were checked for the presence of co-propagating viruses, namely Chronic bee paralysis virus (CBPV), Acute bee paralysis virus (ABPV), Israeli acute paralysis virus (IAPV), Black queen cell virus (BQCV), Slow bee paralysis virus (SBPV) and Sac brood virus (SBV), by qRT-PCR using the protocol and primers detailed in Doublet et al. (2015a). Only a very small amount of CBPV $(<0.001 \%)$ was detected in the DWV inoculum, while the control inoculum was devoid of viruses.
Experimentally injected adult bees were kept in metal cages in an incubator at $+30{ }^{\circ} \mathrm{C}$ for $24 \mathrm{~h}$ to monitor mortality associated with manipulation, which was never found to exceed $10 \%$.

The day after injection, bees ( 2 days old) were subjected to a second infection with $N$. ceranae. Artificial propagations through mass feeding of caged adult honey bees kept in our lab served as a source of $N$. ceranae spores. Spores were counted with a Neubauer haemocytometer under a light microscope $(\times 400)$ and diluted to obtain the required concentration in $50 \%(w / v)$ sucrose solution. As a control inoculum, an extract from uninfected caged honey bees was obtained and subjected to the same procedure as the spore solution. Inocula were prepared freshly the day of experimental infection, as described in Fries et al. (2013). Then they were fed individually with $10 \mu \mathrm{l}$ of $50 \%(w / v)$ sucrose solution containing either $10^{5}$ spores of $N$. ceranae or a control sugar solution devoid of microsporidia. Nosema species identification and absence of spores in the controls were confirmed with a multiplex PCR as described in Fries et al. (2013).

At the end of the experimental set up, all bees had received an injection either with virus solution or control and had been fed individually either with $N$. ceranae spores or control solution. Four treatments were tested: control treatment (C), infection with DWV (D), infection with $N$. ceranae $(\mathrm{N})$ and coinfection with DWV and $N$. ceranae (M). An extra group comprising untreated bees (U) of the same age was included as control for our manipulation procedure (injection and feeding). Up to that point, bees were kept separately depending on their colony of origin and treatment. With the same day of feeding, bees from the three different source colonies that were subjected to the same treatment were equally mixed so as to eliminate any colony genotypic effect. Then bees were individually marked on the thorax with coloured plastic tags (EH Thorne (Beehives) Ltd, UK) numbered 1-99 (different colours for each treatment, total 5 colours), sprayed with sucrose solution and then introduced to the observation hives. Each one of the four hives received 99 bees from each of the five treatments (a total of 495 marked bees per hive). A small coloured dot on the plastic tag was manually added to distinguish bees from different hives and thus to identify bees potentially drifting between hives.

\section{Behavioural observations}

Observations started when bees were 4 days old, leaving 1 day post-introduction to become acclimatised to their new environment. We used scan sampling as described in Kolmes (1984) for recording the behaviour of each marked individual. Three scans per day and hive were conducted, separated by at least a 2 -h interval, between 10 and $16 \mathrm{~h}$ when the colonies were actively foraging. Observations were continued until less than $5 \%$ of the introduced bees from each treatment were 
encountered. When a drifter was encountered, its source colony and behaviour were also recorded. As the hives were located in a room on the sixth floor of a building, observations of nest entrances were not conducted; thus, observations were restricted to within the hive.

\section{Mortality experiment and treatment confirmation}

A mortality assay was set up using an independent subset of newly emerged bees following the same experimental infection procedure described above and using inocula derived from the same propagations as above. Instead of being introduced to observation hives, treated bees were placed in autoclaved metal cages, kept in an incubator $\left(+30{ }^{\circ} \mathrm{C}\right)$ and fed ad libitum with $50 \%(w / v)$ sucrose solution. Each cage contained 21 individual bees that belonged to one of the five treatments described above, namely the untreated group (U), control group (C), N. ceranae-infected group (N), DWVinfected group (D) and DWV $+N$. ceranae (M)-infected group. Each cage was replicated five times. Cages were monitored daily and dead bees were counted and removed. For the $\mathrm{U}$ and $\mathrm{N}$ treatments, only four replicates out of the five were included in the analysis due to leak of sucrose solution (food) in one of the replicate cages that resulted in the death of several bees on 1 day.

Two more replicate cages per treatment were used to verify successful establishment of pathogens and lack of cross contamination across treatments. From these cages, 13-day-old bees were freeze killed in liquid $\mathrm{N}_{2}$ and placed at $-80{ }^{\circ} \mathrm{C}$ for pathogen analysis. Total RNA was extracted from 6 individual bees per treatment per cage using an RNeasy mini kit in a QIAcube robot (Qiagen) according to the manufacturer's instructions. Total cDNA was synthetized using M-MLV Revertase (Promega) following the manufacturer's protocol, using $800 \mathrm{ng}$ of sample RNA. The success of DWV and $N$. ceranae infections was determined by quantitative PCR (Online Resource protocol S1) using specific primers (Online Resource Table S1).

\section{Statistical analysis}

We performed all our statistical analyses using R v3.1.2 (R Core Team 2014).

\section{Behavioural experiment}

We assumed that individuals observed at least once during the experiment were not later rejected. Differences in acceptance rates among the five introduced groups were tested using chisquare test of goodness-of-fit, performed separately for each hive. As the number of individuals drifting across colonies was found to be less than $3.5 \%$ of the total number of individuals accepted per treatment, no statistical analysis of drifting was performed due to inadequate sample size. All identified drifters were removed from any subsequent analysis.

The effect of treatment on the latest age a bee was observed during the experiment was tested using a generalised linear mixed model (GLMM) with Poisson error structure (package 'Ime4', Bates et al. 2014). Treatment was treated as a fixed variable and replicate (host colony) as a random variable. Source colony was not incorporated in the analysis as, after eclosion in the laboratory, bees from the three colonies of origin were equally mixed and not subsequently tracked. The overall effect of treatment was assessed by performing a likelihood test of the full model (fixed plus random factors) against the null model (including only the random factor). The analysis was followed by planned contrasts to test for significant differences between treatment means using the package 'multcomp' (Hothorn et al. 2008), applying a Tukey correction for multiple testing. Planned contrasts involved (i) comparison between the untreated and control groups in order to reveal any effects of manipulation per se and (ii) all possible pairwise comparisons between the control group and infected groups to reveal any effects due to infection. The same procedure was followed to test for differences across different treatments in the mean age of bees performing tasks with clear functional significance related to temporal castes. The tasks 'head in empty/egg cell' (HEC) and 'inspecting larva' (Hlarv) were combined for statistical analysis, as were 'head in cell containing honey (HN) or pollen (HP)' as they represent 'brood care' and 'food processing' tasks, respectively. The same was followed for tasks that indicated foraging activity; 'carrying pollen' (CP), 'attending dance' (AD) and 'performing dance' (PD) were combined.

Differences in the number of tasks performed by each treatment were tested using a Kruskal-Wallis nonparametric test followed by a Nemenyi post hoc test with Tukey approximation for pairwise comparisons (package 'PMCMR', Pohlert 2015). Differences in the frequency with which each task was performed across treatment groups were analysed using a GLMM with binomial error structure. Treatment was included as fixed factor, colony as random factor and performance (yes/no) of each task as a response variable. Planned comparisons as described above were performed to test for differences between groups.

\section{Mortality experiment}

Survival was analysed using a mixed effects Cox proportional hazard model (R package 'survival', Therneau and Grambsch 2000) and the R package 'coxme' (Therneau et al. 2003), with treatment as a fixed factor and cage (replicate) as a random factor. Post hoc analysis to test for significance of differences between treatments was performed using the $\mathrm{R}$ package 
'multcomp' (Hothorn et al. 2008), using Bonferroni correction to account for multiple testing.

\section{Results}

\section{Mortality experiment}

Median survival time $\left(\mathrm{LT}_{50}\right)$ in days $(95 \% \mathrm{CI})$ for each treatment was as follows: $\mathrm{U}=29.5(28-31), \mathrm{C}=26(23-28), \mathrm{N}=23$ (21-25), $\mathrm{D}=17$ (16-18) and $\mathrm{M}=15$ (14-16) (see Fig. 1). Survival was significantly reduced in treatments that contained the virus compared to the control (C vs. D: $z=-4.16, p<0.001$; $\mathrm{C}$ vs. M: $z=-5.62, p<0.001$; Fig. 1). Furthermore, mixed infection was significantly more virulent than $N$. ceranae alone (N vs. M: $z=-3.44, p<0.01$; Fig. 1) but not than DWV alone (D vs. M: $z=-1.54, p>0.1$; Fig. 1). Survival upon $N$. ceranae infection was intermediate between the control and DWV-treated individuals, albeit differences were not statistically significant (C vs. N: $z=-1.97, p>0.1 ; \mathrm{N}$ vs. D: $z=-2.02$, $p>0.1$; Fig. 1). Manipulations did not significantly reduce the survival of bees (U vs. C: $z=-0.94, p>0.1$; Fig. 1).

A post hoc qRT-PCR screen of a subsample of bees collected at 13 days of age showed that all virus-injected (D and M) screened bees ( $n=10$ and $n=11$, respectively) contained DWV $(\mathrm{Cq}$ values $<14)$, while they were free of $N$. ceranae (Fig. S1a). N. ceranae-fed bees ( $\mathrm{N}$ and $\mathrm{M}, n=9$ and $n=11$, respectively) were found to contain high amounts of microsporidian RNA $(\mathrm{Cq}<33)$ except one bee from the $\mathrm{M}$ treatment (Fig. S1b). Two $\mathrm{N}$ treatment bees contained very low levels of DWV $(\mathrm{Cq}>34.5)$, representing background contamination, while control $(\mathrm{C})$ bees $(n=11)$ were devoid of both $N$. ceranae and DWV (Fig. S1).

\section{Behavioural experiment}

The proportion of accepted bees did not differ significantly among the introduced groups in three out of four hives $\left(\chi^{2}(4)=0.699, \chi^{2}(4)=6.852, \chi^{2}(4)=4.0985, p>0.1\right.$ in all cases), with the proportion of bees observed at least once being generally high $(83.8 \% ; 95 \% \mathrm{CI} \pm 5.2 \%)$. Acceptance was much lower in the fourth hive and differed significantly across treatment groups $\left(\chi^{2}(4)=85.318, p<0.001\right)$. However, there was no pattern in acceptance with respect to infection but rather to manipulation (i.e. injection/feeding) (accepted: $\mathrm{U}=$ $91 \%, \mathrm{C}=27 \%, \mathrm{~N}=62 \%, \mathrm{D}=22 \%, \mathrm{M}=20 \%$ ). The proportion of drifters per treatment across the four colonies was (mean $\pm 95 \% \mathrm{CI}$ ) $\mathrm{U}=3.1 \pm 2 \%, \mathrm{C}=3.4 \pm 2 \%, \mathrm{~N}=2.2 \pm 1.1 \%$, $\mathrm{D}=1.3 \pm 1.6 \%$ and $\mathrm{M}=1.9 \pm 2.1 \%$.

The maximum observed age reached by each group (mean in days, $95 \% \mathrm{CI}$ in parentheses: $\mathrm{U}=14.7(14-15.3) ; \mathrm{C}=8.2$ (7.7-8.8); $\mathrm{N}=7.5$ (7.1-7.9); $\mathrm{D}=6.15$ (5.9-6.4); $\mathrm{M}=5.7$ (5.4 5.9)) was found to be significantly affected by treatment (likelihood ratio $\left.\chi^{2}(4)=1781.9, p<0.001\right)$. Post hoc analysis showed that the virus-treated individuals ( $\mathrm{D}$ and $\mathrm{M}$ ) were observed for a significantly shorter period than the $N$. ceranae (N)-treated group (D vs. N; M vs. N: $p<0.001$ for both comparisons), which in turn were observed for a significantly shorter period than the control (C) group (N vs. C; D vs. C; $\mathrm{M}$ vs. C: $p<0.001$ all comparisons). Manipulation itself was found to lower the maximum observed age, too (U vs. C; $p<0.01)$. No significant difference was observed between

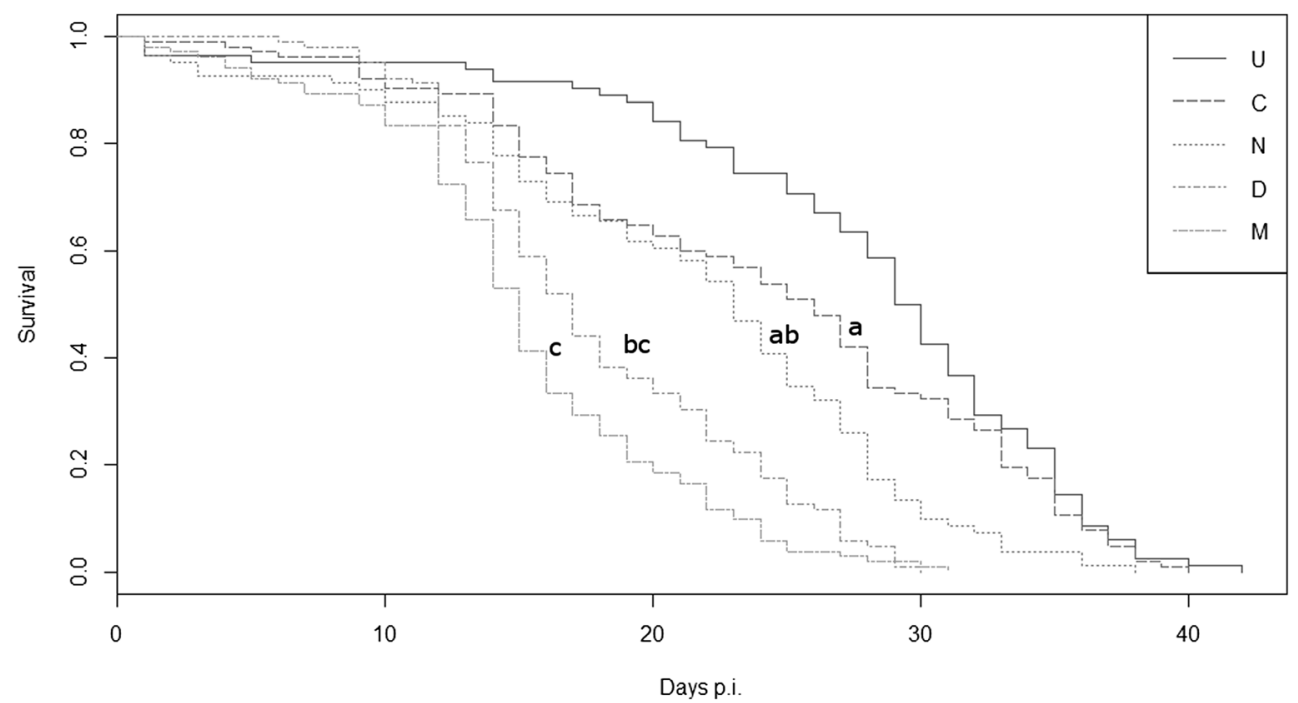

Fig. 1 Fitted Cox proportional hazard survival curves in days postinfection (p.i.). Treatments tested: $U=$ untreated, $C=$ control, $N=$ $N$. ceranae, $D=\mathrm{DWV}, M=\operatorname{mix}(\mathrm{DWV}+N$. ceranae $)$. Different lowercase letters indicate significant difference at $p<0.05$ (post hoc pairwise planned comparisons, Bonferroni corrected). Manipulation per

se did not significantly reduce survival of bees (U vs. C: $p>0.1$; comparison not shown in the figure). Note that survival plots are for visualisation only as they do not incorporate random effects (replicate cages) 
Table 1 List of behavioural tasks observed (see Kolmes (1984) for a more detailed description of each task)

\begin{tabular}{|c|c|c|}
\hline Task & Code & Description \\
\hline Into empty/egg cell & HEC & Enters a cell that is either empty or contains an egg \\
\hline Inspect/feed larva & Hlarv & Inserts its head into a cell containing a larva \\
\hline Into honey cell & $\mathrm{HN}$ & Inserts its head into or enters a cell containing honey \\
\hline Into pollen cell & HP & Inserts its head into or enters a cell containing stored pollen \\
\hline Building comb & $\mathrm{BC}$ & $\begin{array}{l}\text { Manipulates wax in areas where cells are in construction } \\
\text { using mouthparts }\end{array}$ \\
\hline Attend dance & $\mathrm{AD}$ & Follows a dancing worker \\
\hline Dance & $\mathrm{PD}$ & Dances with or without pollen \\
\hline Walk with pollen & $\mathrm{CP}$ & Has pollen in her pollen baskets \\
\hline Mouth wax-brood & $\mathrm{TB}$ & Trims/smooths wax cappings on brood cells or sealed brood \\
\hline Mouth wax-honey/pollen & $\mathrm{TH}$ & $\begin{array}{l}\text { Trims/smooths wax cappings on cells containing honey/pollen } \\
\text { or capping them }\end{array}$ \\
\hline Attend queen & AQ & Is a member of the queen retinue \\
\hline Antennal contact with worker & ANT & Is in mutual contact with another worker \\
\hline Fan wings & FAN & Produces an air current with her wings \\
\hline Feeding worker & FW & The proboscis is extended between another bee's mandibles \\
\hline Being fed & GF & $\begin{array}{l}\text { The proboscis of another worker is extended between the } \\
\text { mandibles of the worker }\end{array}$ \\
\hline Being groomed & GG & Stands and is cleaned by another worker \\
\hline Grooming self & GS & Grooms a part of its own body \\
\hline Grooming worker & GW & Grooms another worker bee \\
\hline Lateral shake & $\mathrm{CD}$ & Rapidly shakes its body from side to side (cleaning dance) \\
\hline Chew on hive & $\mathrm{CH}$ & $\begin{array}{l}\text { Uses its mandibles to chew on the inside of the hive } \\
\text { (e.g. wooden frames, etc.) }\end{array}$ \\
\hline Standing & ST & Stands motionless (lack of activity) \\
\hline Walk & WALK & Walks inside the hive \\
\hline
\end{tabular}

individuals treated with DWV or a mix of DWV $+N$. ceranae (D vs. M: $p>0.05$ ). A histogram of the distribution of observed behavioural acts over the age (in days) of marked bees across treatments is given in Online Resource Fig. S2.

In total, 22 different behavioural tasks were recorded (Table 1) across a total of 11,566 behavioural acts. Individuals injected with DWV (in single or in mixed infection with $N$. ceranae) tended to perform a slightly smaller range of tasks per colony than bees not injected with DWV. However, differences were not significant (Kruskal-Wallis test, $p>0.05$ in all pairwise comparisons; mean number $( \pm \mathrm{SE})$ of tasks performed by each treatment group per colony: $\mathrm{U}=21.5 \pm 0.3 ; \mathrm{C}=$ $17.3 \pm 1.9 ; \mathrm{N}=19.3 \pm 1.3 ; \mathrm{D}=16.3 \pm 0.5 ; \mathrm{M}=15.3 \pm 1.2$ ), indicating that repertoire size was not affected markedly by infection. Irrespective of the repertoire size, several behavioural tasks were never observed in some treatments across the four colonies. Specifically, DWV-injected bees were never observed grooming their nestmates (behaviour GW), while Mtreated bees were never observed to attend the queen (behaviour AQ), feed another worker (behaviour FW) or trim wax capping on cells containing honey (behaviour TH) (Fig. 2). However, behaviours GW, AQ and FW were rarely observed across all treatments (relative frequency $<1.2 \%$; Fig. 2).
As expected, worker honey bees progressed through the expected age polyethism schedule and were found to perform tasks related to brood care (behaviours HEC + Hlarv) earlier in their lives and then to move to tasks related to food processing $(\mathrm{HN}+\mathrm{HP})$, comb building (MC) and foraging (AD + PD + $\mathrm{CP}$ ) later in their lives. However, these temporal patterns were found to be skewed towards a younger age in experimental treatments (Fig. 3). Pathogen-treated bees tended to perform tasks earlier in life than control bees. Virus-treated individuals (D and $\mathrm{M}$ ) exhibited even lower mean ages in performing a specific behaviour than N. ceranae-treated bees (Fig. 3). Specifically, there was a significant main effect of treatment on brood care tasks (likelihood ratio $\chi^{2}(4)=441.6, p<0.001$; Fig. 3), food processing tasks (likelihood ratio $\chi^{2}(4)=254.8$, $p<0.001$; Fig. 3) and comb building $\left(\chi^{2}(4)=132.6, p<0.05\right.$; Fig. 3). Even if manipulation per se was found to lower significantly the age when bees performed these tasks (U vs. C, $p<0.05$; Fig. 3), pathogen-treated individuals performed these tasks at an even earlier age than the control group (N, D, M vs. C: all $p<0.05$; Fig. 3). Differences between virus-treated (D and $\mathrm{M}$ ) and $N$. ceranae-treated bees were statistically significant in the case of food processing tasks, which were performed earlier by virus-treated (D and $\mathrm{M}$ ) than $N$. ceranae- 


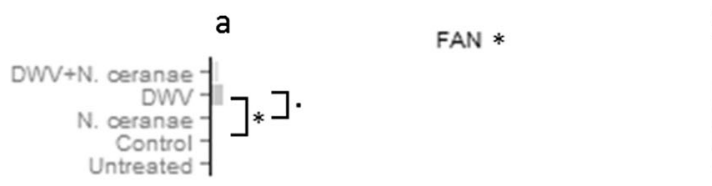

GG*
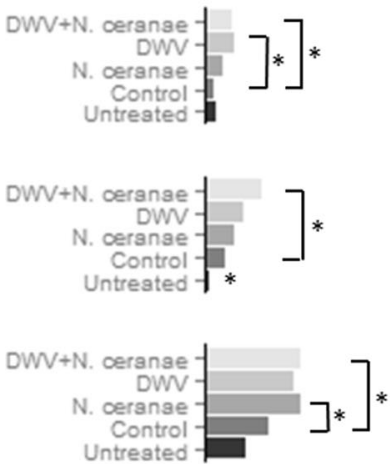

ANT *

Foraging *

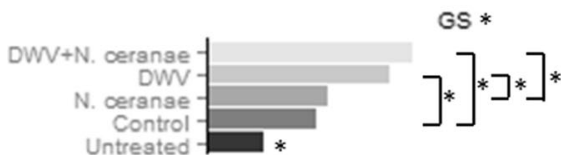

b
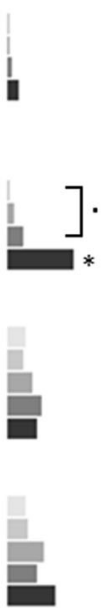

$A Q$

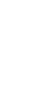

$\mathrm{TH} *$

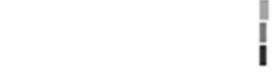

$\mathrm{CH}$

ST *

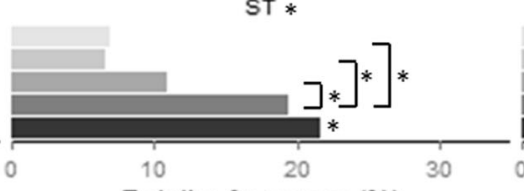

Relative frequency (\%)

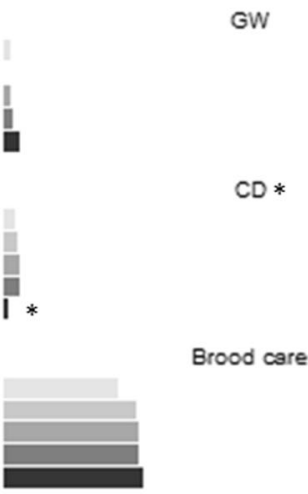

C

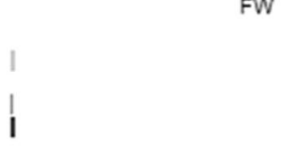

GF
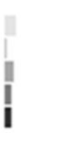

ow

$C D *$

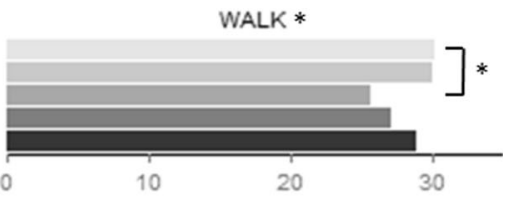

Fig. 2 Relative frequencies (\%) of each behavioural task performed (brood care: HEC + Hlarv; food processing: $\mathrm{HN}+\mathrm{HP}$; foraging: $\mathrm{CP}+$ $\mathrm{AD}+\mathrm{PD}$ ) per treatment. Tasks were sorted into three groups: tasks with higher relative frequency in the pathogen-treated compared to control bees (a, left column); tasks with lower relative frequency in the pathogen-treated compared to control bees (b, middle column); tasks with no difference in the relative frequency between pathogen treated and control bees (c, right column). Stars in panel header indicate a significant main effect of treatment (GLMM, $p<0.05)$. Significant pairwise comparisons are also shown $(p<0.05$, Tukey correction for multiple testing). Dots in pairwise comparisons indicate marginal significant differences ( $p$ values $<0.07$ ). Stars next to the untreated group indicate a significant difference compared to the control group $(p<0.05)$. See Table 1 for task abbreviations treated individuals (D vs. N, M vs. N: both $p<0.05$; D vs. M: $p>0.1$; Fig. 3). Differences in the timing of brood care and comb building did not differ between virus treatments ( $\mathrm{D}$ and $\mathrm{M}$ ) and N. ceranae treatments (D vs. N, M vs. N, D vs. M: all $p>0.1$; Fig. 3).

Foraging, which was estimated indirectly by pooling foraging-related activities (i.e. behaviours $\mathrm{AD}, \mathrm{CP}$ and $\mathrm{PD}$ ), was also affected by treatment $\left(\chi^{2}(4)=34.2, p<0.001\right)$, with mixed pathogen-treated individuals commencing foraging significantly earlier compared to control or $N$. ceranae-treated bees (M vs. C, M vs. N: both $p<0.05$; Fig. 3). Single virustreated bees exhibited an intermediate age compared to the mix and N. ceranae group (D vs. M, D vs. N: both $p>0.05$; D vs. C: $p<0.05$; Fig. 3$)$. N. ceranae-treated bees also tended to start foraging earlier than control bees, but the difference was not statistically significant (N vs. C: $p>0.1$; Fig. 3). Manipulation was not found to have an effect on age of foraging (U vs. C: $p>0.1$; Fig. 3 ).

The intensity with which several tasks were performed was found to be significantly affected by treatment. There was a general trend for pathogen-treated bees $(\mathrm{D}, \mathrm{N}$ or $\mathrm{M})$ to engage more frequently than control bees in fanning (FAN), being groomed (GG), self-grooming (GS), being in antennal contact with other nestmates (ANT), trimming wax capping on brood cells (TB) and foraging (Fig. 2a). The most pronounced differences were found in aspects of grooming behaviour. Individuals treated with DWV (alone or mixed with $N$. ceranae) were groomed by their nestmates or groomed themselves significantly more often compared to control bees (GG and GS acts: D vs. C, M vs. C: both $p<0.05$; Fig. $2 \mathrm{a}$ ) or, in the latter case, even more often than individuals fed only $N$. ceranae (behaviour GS: D vs. N, M vs. N: both $p<0.05$; Fig. 2a). Moreover, $N$. ceranae-treated individuals (single infection or mixed with DWV) more often engaged in antennal contact with their nestmates compared to control bees (ANT: $\mathrm{N}$ vs. C, M vs. C; both $p<0.05$; Fig. 2a). Finally, the frequency of foraging (behaviours $\mathrm{CP}+\mathrm{AD}+\mathrm{PD}$ ) was also found to increase in pathogen treatments, with mix-treated individuals foraging significantly more often than control bees (M vs. C: $p<0.05$; Fig. 2a). Manipulation per se was also found to 


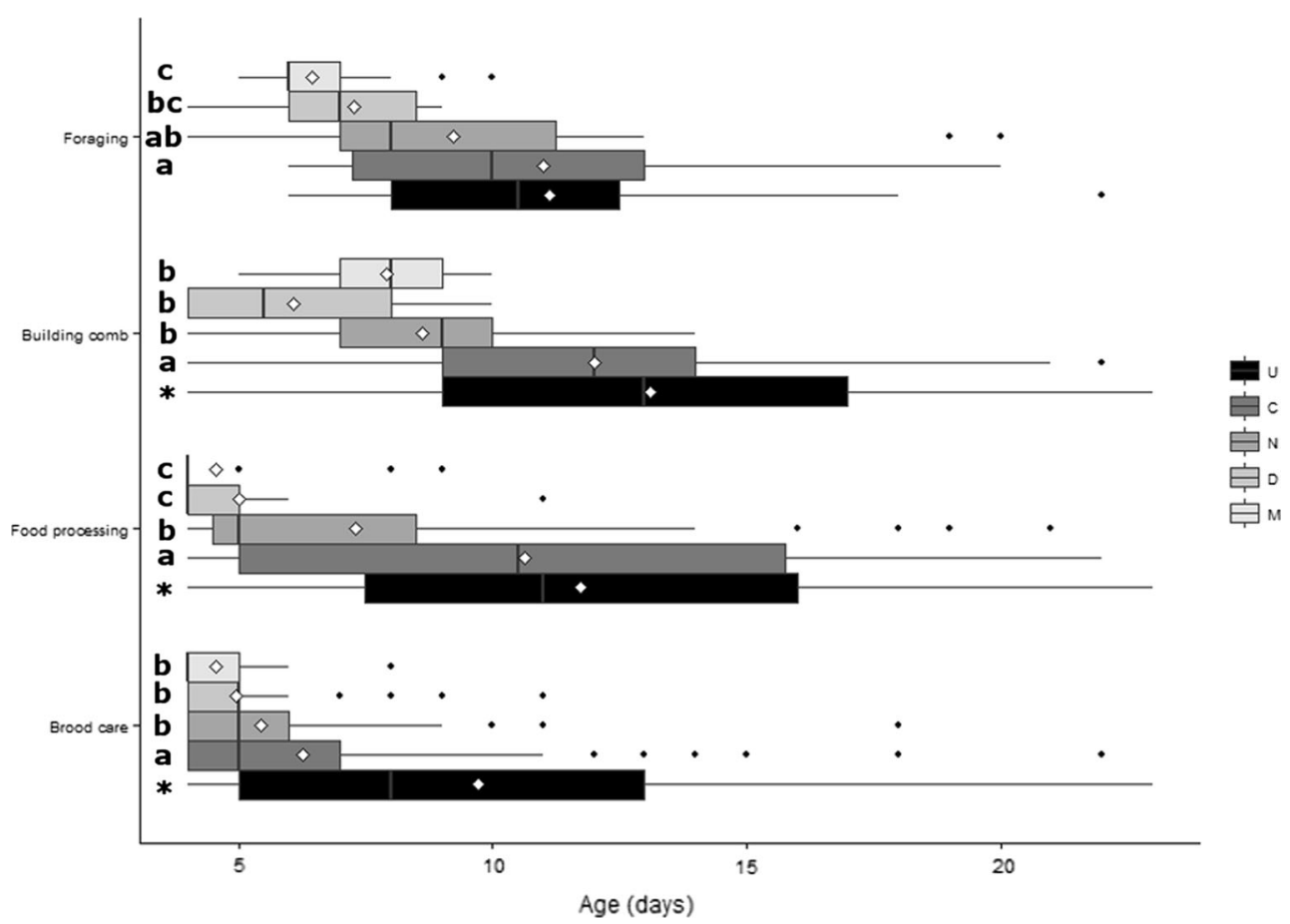

Fig. 3 Box-plot showing the median age of bees performing tasks related to temporal patterns by treatment group ( $U=$ untreated, $C=$ control, $N=$ $N$. ceranae, $D=\mathrm{DWV}, M=\mathrm{DWV}+N$. ceranae). Diamonds indicate the mean of each group, while black dots indicate outliers. For 'brood care', the tasks HEC and Hlarv were combined; for 'food processing', HN and $\mathrm{HP}$ were combined; and for 'foraging', $\mathrm{AD}, \mathrm{PD}$ and $\mathrm{CP}$ were combined (for abbreviations of behaviours, see Table 1). Different lowercase letters indicate significant differences at $p<0.05$. Asterisks $(*)$ indicate significant difference between untreated $(U)$ and control $(C)$ groups at $p<0.05$. Pairwise planned comparisons (see "Material and methods" section) were performed within each behavioural task increase significantly the frequency of self-grooming (GS), trimming wax capping of brood cells (TB) and foraging ( $\mathrm{U}$ vs. C; $p<0.05$; Fig. 2a).

The frequency with which other tasks (i.e. attending the queen (AQ), trimming wax cappings on cells with honey (TH), chewing on hive (CH), building comp (BC), food processing and lack of activity (ST)) was undertaken tended to be reduced under pathogen treatment and more pronounced with DWV (alone or mixed with $N$. ceranae) than $N$. ceranae (Fig. 2b). However, pairwise comparisons were not statistically significant, except in the cases of food processing-related tasks (behaviours HN $+\mathrm{HP}$ ) and lack of activity (behaviour ST). Mixed infected bees were found to perform food processing-related tasks (behaviours $\mathrm{HN}+\mathrm{HP}$ ) less often than control bees (M vs. C: $p<0.05$; Fig. $2 b$ ), indicating that the relative amount of work performed was not equally distributed across treatment groups. Most noticeably, infected bees spent less time standing inactive in the hive compared to control bees (behaviour ST: C vs. N, C vs. D, C vs. M; all $p<0.05$; Fig. 2b), with DWV-treated individuals (single infection or mixed with $N$. ceranae) exhibiting even less inactivity than $N$. ceranae-treated bees ( $\mathrm{N}$ vs. D, N vs. M; both $p<0.05$; Fig. 2b). The intensity with which the remaining tasks were performed was found to exhibit little or no variation across treatments (Fig. 2c).

\section{Discussion}

Honey bees within a hive react to a wide range of stressors that can affect their behaviour (Schulz et al. 1998; Schneider et al. 2012; Dussaubat et al. 2013). Here we demonstrate a direct link between experimental exposure to pathogens, with distinct pathologies and a more rapid rate of behavioural maturation of honey bee workers within a common hive environment. This was reflected in changes in the pace that bees passed through the temporal polyethism schedule while at the same time maintaining the same pattern of temporal changes. This accelerated pace was more pronounced for bees treated with the more virulent pathogen in terms of hostinduced mortality (Fig. 3), namely DWV versus $N$. ceranae (Figs. 1 and S2). Co-infection did not show any additive effect, even if in some cases it tended to slightly magnify the effect of DWV (Fig. 3).

An accelerated pace of behavioural transition can have adaptive value for the colony as it results in infected bees moving away from the central area of the colony, where the brood and young workers are found, and towards the periphery, where foragers and older workers are present (Seeley 1982; Winston 1987). Infestation of the central area of the colony can be fatal because this is where the sensitive brood and queen reside, and thus, the speeding up of behavioural 
ageing can be a prophylactic measure to limit contact rates between infected bees and brood or the queen, supporting the conveyor belt model of Schmid-Hempel (1998).

Rapid behavioural maturation leading to precocious foraging has been associated with reduced lifespan, either due to infection or injury (Tofilski 2009; Woyciechowski and Moroń 2009). According to the division of risk hypothesis, individuals with reduced lifespan tend to adjust their behavioural schedule by performing risky tasks (i.e. precocious foraging) earlier in life compared to healthy individuals as their death is less costly to the colony (Woyciechowski and Kozłowski 1998; Tofilski 2006). In accordance, we find that results from our cage mortality experiments correspond to the onset of foraging, suggesting that parasites could induce precocious foraging by decreasing survival of bees. Virus-treated individuals exhibited both the lowest rates of survival and the earliest age at onset of foraging, while $N$. ceranae-fed bees showed intermediate patterns to control and virus-treated individuals for both mortality and onset of foraging (Figs. 1 and 3). Apart from reduced lifespan, nutritional status has also been found to be an important predictor dictating age at first foraging, with starvation being associated with precocious foraging (Schulz et al. 1998; Toth and Robinson 2005). In terms of parasitism, energetic stress is considered to be one of the main symptoms of infection by $N$. ceranae (Mayack and Naug 2009), but studies have yet to addressed the effects of DWV on the nutritional status of infected individuals.

Whether accelerated maturation represents an act of 'adaptive suicide' to protect nestmates (Smith Trail 1980) or a parasite manipulation of host behaviour is difficult to demonstrate. An accelerated pace of behavioural transition and precocious foraging can be beneficial for the pathogen itself as it can facilitate between-colony and even between-species horizontal transmission, a necessary step in the life of many honey bee pathogens (Fries and Camazine 2001). Goblirsch et al. (2013) showed that $N$. ceranae disrupts underlying honey bee physiology. More specifically, using both cage and field experiments, Goblirsch et al. (2013) showed a link between N. ceranae infection and alterations in the vitellogenin ( $\mathrm{Vg}$ )-juvenile hormone $(\mathrm{JH})$ regulatory network, which is known to influence the timing of switching from nurse to foraging bee (Robinson 1987; Nelson et al. 2007). Whether DWV is able to alter the same pathway has not been shown yet, but its presence and replication in the fat body and haemolymph (Fievet et al. 2006; Boncristiani et al. 2009), where $\mathrm{Vg}$ and $\mathrm{JH}$ are found (Corona et al. 2007), suggests that this may be the case. Moreover, precocious foraging (Janmaat and Winston 2000) and lower Vg levels (Amdam et al. 2004) have been found in bees parasitized by the mite $V$. destructor, which is known to vector and boost replication of DWV (Shen et al. 2005). A study by McDonnell et al. (2013) suggests that the transcriptomic profiles in the brain of bees parasitized by either $N$. ceranae or Varroa mites and thus DWV (revealed by post hoc analysis of mite-infested bees) share more similarities compared to healthy individuals, either nurses or foragers. They argue for the idea that infected individuals are not true foragers but exhibit precocious foraging as an altruistic act of self-removal. Moreover the fact that immune-challenged individuals exhibit a similar profile to 'normal' foragers (Alaux et al. 2012) indicates that the unique brain profile observed in $N$. ceranae and Varroa (and thus DWV)-infected individuals is not a mere consequence of immune stimulation but rather may be pathogen driven.

Interestingly, we found infection to decrease the time individuals spend resting. This result is in contrast with two previous studies (Annoscia et al. 2015; Retschnig et al. 2015) that found the opposite for bees parasitized by either $N$. ceranae or Varroa mites; increased 'laziness' in the case of $N$. ceranaeinfected bees was attributed by Retschnig et al. (2015) to energetic stress imposed by the microsporidian (Mayack and Naug 2009). However, energetic stress due to $N$. ceranae infection has been associated with longer foraging trips and reduced time spent within the colony (Alaux et al. 2014; Naug 2014), which could better explain the lower frequency of resting in pathogen-treated individuals within the colony. Moreover, in Drosophila melanogaster, altered energy homeostasis upon food deprivation has been found to result in increased locomotion (Lee and Park 2004; Yang et al. 2015); food deprivation was associated with octopamine production, which acts as a neurohormone in the brain (Farooqui et al. 2004) and plays a critical role regulating starvation hyperactivity (Yang et al. 2015). Increased octopamine titre due to parasitic infection has been shown in several invertebrates (Adamo 2002), while bees injected with octopamine increase grooming and foraging (Fussnecker et al. 2006). Upregulation of octopamine pathways has recently been found in $N$. ceranae-infected foragers in the field (Mayack et al. 2015), but the effects of DWV on the energetic status of bees or on octopamine physiological pathways are still unknown. In our study, the increased hyperactivity we observed in DWV-treated individuals was not compensated by an increased frequency in random walking but rather by increased grooming and frequency of foraging. Moreover, DWV-treated individuals avoided contact with food storage cells and exhibited reduced involvement in food processing tasks. These observations suggest altered octopamine regulation and starvation hyperactivity in search of sources of food outside the nest to meet their energy demands, minimising the risk of contamination of nest food stores. However, because foraging was only estimated indirectly in our study, we can only speculate about outside-nest behaviours. Determining the amount of food DWV-infected bees bring back to the colony will help understand if this increase in foraging frequency is associated with less effective food recruitment, as was shown in N. ceranae-infected bees (Naug 2014).

We also found treatment with DWV to increase the frequency of social activities related to grooming behaviour 
(Fig. 2). Specifically, we found DWV-infected individuals to exhibit increased self-grooming activity and to be more frequently cleaned by their nestmates while at the same time they were never observed to clean their nestmates (Fig. 2). Grooming is a well-developed behavioural trait in eusocial insects and plays an important part in social immunity (Cremer et al. 2007), serving both individual (i.e. hygiene) and colony (i.e. maintenance of colony odour homeostasis) functions (Crozier and Dix 1979; Zhukovskaya et al. 2013).

Grooming is usually an effective act against removal of external pathogens such as fungi or ectoparasites (SchmidHempel 1998; Reber et al. 2011). Thus, it is quite surprising that we observed an increase grooming in virus-treated individuals. A possible explanation could be that DWV-infected individuals are able to perceive their health status and try to prevent a secondary infection that will reduce their lifespan further. Nestmates in turn seem to be also able to recognise DWV-infected individuals and increase grooming of them (allogrooming). This could potentially be part of the strategy of a virus to increase its transmission within a colony. During allogrooming, groomers face an increased risk of infection as they can be exposed to viral particles (e.g. through licking). However, the degree of disease spread in colonies due to allogrooming has been found to strongly depend on the infectiousness of the pathogen, at least in ant colonies (Theis et al. 2015), information which is not yet known for DWV. However, bees immune stimulated with bacteria were also found to be the target of increased grooming, including antennal contact, probably resulting from changes in the cuticular hydrocarbon (CHC) profile of infected bees (Richard et al. 2008, 2012), which serves as an olfactory recognition cue among nestmates (Breed 1983). Immunosuppression and changes in the CHC profile of bees infected by DWV, $N$. ceranae and Varroa mites have been previously demonstrated (Baracchi et al. 2012; Nazzi et al. 2012; McDonnell et al. 2013). That we found significant changes only in bees that we treated with DWV may indicate that the strength of the signal perceived by the nestmates, or the infected individual itself and thus its change in behavioural response, depends on the virulence of the pathogen.

Parasitic infection might be expected to decrease contacts between nestmates as contact facilitates transmission (Schmid-Hempel 1998; Cremer et al. 2007). However, in our study, pathogen treatment was found to increase the frequency of antennal contacts, which were more pronounced in $N$. ceranae-treated individuals (Fig. 2) than DWV-treated bees. A possible explanation could be that $N$. ceranae-infected bees may solicit for more food due to their lower energetic state. However, previous studies have not shown any significant alteration in social interactions, including grooming or antennal contact, of bees infected with $N$. ceranae (Retschnig et al. 2015) or Varroa mites/DWV (McDonnell et al. 2013). But differences in samples sizes, observation and/or infection methodology may account for inconsistencies across studies. Regarding increased infection risk, it would now be interesting to investigate didactic interactions, namely to determine whether any type of increased contact frequency (i.e. grooming, antennal contact) was performed among pathogen-treated individuals or between treated and untreated nestmates and leads to pathogen transmission. Application of automated tracking systems (e.g. Mersch et al. 2013; Gernat et al. 2015) that monitor all bees continuously within a hive will allow future studies to shed light onto those interactions.

The findings of our study also suggest that stress during manipulation procedures can alter bee behaviour, though to a lesser extent compared to parasitic infection (Fig. 2). This is not surprising given that injection is a process that cannot only place a stress on bees but can also cause non-pathogenic immune stimulation (Evans et al. 2006; Siede et al. 2012), affecting subsequent honey bee behaviour (e.g. Alaux et al. 2014).

In summary, we find that pathogen pressure in honey bees does not result in major differences in the pattern of the temporal polyethism schedule but rather to an accelerated progression through it, a novel finding especially concerning DWV. The maintenance of the pattern of temporal polyethism indicates that task transitions are linked and cannot be easily dissociated from each other. Moreover, pathogen-treated individuals, even if they were able to contribute to colony needs, did not seem to compensate for their reduced time spent as members of the colony with an increasing frequency of within-hive activities. Instead, they allocated more time to grooming and foraging compared to control bees. To what extent accelerated task transitioning is a strategy adopted by individual bees to protect the colony from disease is unknown. Specifically, it remains to be investigated exactly how acceleration of behavioural transitions influences disease dynamics, particularly in terms of transmission within the colony. As complete social isolation from the brood or food storage areas was not observed in either of the treatments, it seems that there is a trade-off between behavioural mechanisms reducing infection risk and performing colony maintenance duties for the fitness benefits of the colony. Regarding hygiene-related behaviours, increased self-grooming has been previously described in fungus-infected ants (Reber et al. 2011), and thus, the observed patterns may be generally applicable to eusocial Hymenoptera. Finally, our results also suggest that the strength of behavioural shifts depends on the identity of the pathogen. More benign parasites such as the microsporidian N. ceranae (Fries and Camazine 2001) may have a less pronounced effect on behaviour compared to more virulent pathogens such as DWV. To fully understand the adaptive nature of these responses and the general role of pathogen pressure in regulating social behaviours, studies focusing on multiple pathogens across a wider range of eusocial insects are required. 
Acknowledgments We thank Vincent Doublet and Panagiotis Theodorou for technical support and for their useful comments on the manuscript. We also thank the editor and two anonymous reviewers for their comments, which helped improve the manuscript. This work was supported by the Federal Ministry of Food, Agriculture and Consumer Protection (Germany): Fit Bee project (grant 511-06.01-28-1-71.007-10) and the Insect Pollinators Initiative (IPI grant BB/1000100/1). The IPI is funded jointly by the Biotechnology and Biological Sciences Research Council, the Department for Environment, Food and Rural Affairs, the Natural Environment Research Council, The Scottish Government and The Wellcome Trust, under the Living with Environmental Change Partnership.

Compliance with ethical standards All experiments conducted and presented in the manuscript comply with the laws and rules of the institution and country in which they were performed.

Conflict of interest The authors declare that they have no competing interests.

\section{References}

Adamo SA (2002) Modulating the modulators: parasites, neuromodulators and host behavioral change. Brain Behav Evol 60:370-377. doi:10.1159/000067790

Alaux C, Crauser D, Pioz M, Saulnier C, Le Conte Y (2014) Parasitic and immune-modulation of flight activity in honey bees tracked with optical counters. J Exp Biol 217:3416-3424. doi:10.1242/jeb. 105783

Alaux C, Kemper N, Kretzschmar A, Le Conte Y (2012) Brain, physiological and behavioral modulation induced by immune stimulation in honeybees (Apis mellifera): a potential mediator of social immunity? Brain Behav Immun 26:1057-1060. doi:10.1016/j.bbi.2012. 04.004

Amdam GV, Hartfelder K, Norberg K et al (2004) Altered physiology in worker honey bees (Hymenoptera: Apidae) infested with the mite Varroa destructor (Acari: Varroidae): a factor in colony loss during overwintering? J Econ Entomol 97:741-747. doi:10.1603/00220493(2004)097[0741:APIWHB]2.0.CO;2

Annoscia D, Del Piccolo F, Covre F, Nazzi F (2015) Mite infestation during development alters the in-hive behaviour of adult honeybees. Apidologie 46:306-314. doi:10.1007/s13592-014-0323-0

Bailey L, Fernando EFW (1972) Effects of sacbrood virus on adult honey-bees. Ann Appl Biol 72:27-35. doi:10.1111/j.1744-7348.1972. tb01268.x

Baracchi D, Fadda A, Turillazzi S (2012) Evidence for antiseptic behaviour towards sick adult bees in honey bee colonies. J Insect Physiol 58:1589-1596. doi:10.1016/j.jinsphys.2012.09.014

Bates D, Mächler M, Bolker B, Walker S (2014) lme4: linear mixedeffects models using Eigen and S4. R package version 1.1-8, http://CRAN.R-project.org/package=lme4

Beshers S, Fewell J (2001) Models of division of labor in social insects. Annu Rev Entomol 46:413-440. doi:10.1146/annurev.ento.46.1. 413

Boncristiani HF, Di Prisco G, Pettis JS et al (2009) Molecular approaches to the analysis of Deformed wing virus replication and pathogenesis in the honey bee, Apis mellifera. Virol J 6:221. doi:10.1186/1743422X-6-221

Breed M (1983) Nestmate recognition in honey bees. Anim Behav 31: 86-91. doi:10.1016/S0003-3472(83)80176-6
Carreck NL, Ball BV, Martin S (2010) Honey bee colony collapse and changes in viral prevalence associated with Varroa destructor. J Apic Res 49:93-94. doi:10.3896/IBRA.1.49.1.13

Chen Y, Siede R (2007) Honey bee viruses. Adv Vir Res 70:33-80. doi: 10.1016/S0065-3527(07)70002-7

Corona M, Velarde RA, Remolina S et al (2007) Vitellogenin, juvenile hormone, insulin signaling, and queen honey bee longevity. Proc Natl Acad Sci U S A 104:7128-7133. doi:10.1073/pnas. 0701909104

Cox-Foster DL, Conlan S, Holmes EC et al (2007) A metagenomic survey of microbes in honey bee colony collapse disorder. Science 318: 283-287. doi:10.1126/science. 1146498

Cremer S, Armitage SAO, Schmid-Hempel P (2007) Social immunity. Curr Biol 17:R693-R702. doi:10.1016/j.cub.2007.06.008

Crozier RH, Dix MW (1979) Analysis of two genetic models for the innate components of colony odor in social Hymenoptera. Behav Ecol Sociobiol 4:217-224. doi:10.1007/BF00297645

Dainat B, Evans JD, Chen YP et al (2012) Dead or alive: Deformed wing virus and Varroa destructor reduce the life span of winter honeybees. Appl Environ Microbiol 78:981-987. doi:10.1128/AEM. 06537-11

de Miranda JR, Genersch E (2010) Deformed wing virus. J Invertebr Pathol 103:S48-S61. doi:10.1016/j.jip.2009.06.012

Doublet V, Labarussias M, de Miranda JR et al (2015a) Bees under stress: sublethal doses of a neonicotinoid pesticide and pathogens interact to elevate honey bee mortality across the life cycle. Environ Microbiol 17:969-983. doi:10.1111/1462-2920.12426

Doublet V, Natsopoulou ME, Zschiesche L, Paxton RJ (2015b) Withinhost competition among the honey bees pathogens Nosema ceranae and Deformed wing virus is asymmetric and to the disadvantage of the virus. J Invertebr Pathol 124:31-34. doi:10.1016/j.jip.2014.10. 007

Downey DL, Higo TT, Winston ML (2000) Single and dual parasitic mite infestations on the honey bee, Apis mellifera L. Insectes Soc 47: $171-176$

Dussaubat C, Maisonnasse A, Crauser D et al (2013) Flight behavior and pheromone changes associated to Nosema ceranae infection of honey bee workers (Apis mellifera) in field conditions. J Invertebr Pathol 113:42-51. doi:10.1016/j.jip.2013.01.002

Evans JD, Aronstein K, Chen YP et al (2006) Immune pathways and defence mechanisms in honey bees Apis mellifera. Insect Mol Biol 15:645-656. doi:10.1111/j.1365-2583.2006.00682.x

Evans JD, Schwarz RS (2011) Bees brought to their knees: microbes affecting honey bee health. Trends Microbiol 19:614-620. doi:10. 1016/j.tim.2011.09.003

Evans JD, Spivak M (2010) Socialized medicine: individual and communal disease barriers in honey bees. J Invertebr Pathol 103:S62-S72. doi:10.1016/j.jip.2009.06.019

Farooqui T, Vaessin H, Smith BH (2004) Octopamine receptors in the honeybee (Apis mellifera) brain and their disruption by RNAmediated interference. J Insect Physiol 50:701-713. doi:10.1016/j. jinsphys.2004.04.014

Fievet J, Tentcheva D, Gauthier L et al (2006) Localization of deformed wing virus infection in queen and drone Apis mellifera L. Virol J 3: 16. doi:10.1186/1743-422X-3-16

Free JB, Spencer-Booth Y (1961) Further experiments on the drifting of honey-bees. J Agric Sci 57:153-158

Fries I (2010) Nosema ceranae in European honey bees (Apis mellifera). J Invertebr Pathol 103:S73-S79. doi:10.1016/j.jip.2009.06.017

Fries I, Camazine S (2001) Implications of horizontal and vertical pathogen transmission for honey bee epidemiology. Apidologie 32:199 214. doi:10.1051/apido:2001122

Fries I, Chauzat M-P, Chen Y-PP et al (2013) Standard methods for Nosema research. J Apic Res 52:1-28. doi:10.3896/IBRA.1.52.1.14 
Fürst MA, McMahon DP, Osborne JL et al (2014) Disease associations between honeybees and bumblebees as a threat to wild pollinators. Nature 506:364-366. doi:10.1038/nature12977

Fussnecker BL, Smith BH, Mustard JA (2006) Octopamine and tyramine influence the behavioral profile of locomotor activity in the honey bee (Apis mellifera). J Insect Physiol 52:1083-1092. doi:10.1016/j. jinsphys.2006.07.008

Genersch E, Aubert M (2010) Emerging and re-emerging viruses of the honey bee (Apis mellifera L.). Vet Res 41:54. doi:10.1051/vetres/ 2010027

Gernat T, Rao V, Deng W et al (2015) Temporal correlations not network structure, promote information flow in honey bee social networks. Central European Section Meeting of the International Union for the Study of Social Insects (USSI). Lichtenfels, Germany, p 20

Goblirsch M, Huang ZY, Spivak M (2013) Physiological and behavioral changes in honey bees (Apis mellifera) induced by Nosema ceranae infection. PLoS One 8, e58165. doi:10.1371/journal.pone.0058165

Gordon D (1996) The organization of work in social insect colonies. Nature 380:121-124. doi:10.1038/380121a0

Hart BL (1990) Behavioral adaptations to pathogens and parasites: five strategies. Neurosci Biobehav Rev 14:273-294. doi:10.1016/ S0149-7634(05)80038-7

Higes M, García-Palencia P, Martín-Hernández R, Meana A (2007) Experimental infection of Apis mellifera honeybees with Nosema ceranae (Microsporidia). J Invertebr Pathol 94:211-217. doi:10. 1016/j.jip.2006.11.001

Hothorn T, Bretz F, Westfall P (2008) Simultaneous inference in general parametric models. Biometrical J 50:346-363

Huang ZY, Robinson GE (1992) Honeybee colony integration: workerworker interactions mediate hormonally regulated plasticity in division of labor. Proc Natl Acad Sci U S A 89:11726-11729. doi:10. 1073/pnas.89.24.11726

Huang ZY, Robinson GE (1996) Regulation of honey bee division of labor by colony age demography. Behav Ecol Sociobiol 39:147158. doi:10.1007/s002650050276

Iqbal J, Mueller U (2007) Virus infection causes specific learning deficits in honeybee foragers. Proc R Soc B 274:1517-1521. doi:10.1098/ rspb.2007.0022

Janmaat AF, Winston ML (2000) The influence of pollen storage area and Varroa jacobsoni Oudemans parasitism on temporal caste structure in honey bees (Apis mellifera L.). Insectes Soc 47:177-182. doi:10. 1007/PL00001698

Johnson BR (2008) Within-nest temporal polyethism in the honey bee. Behav Ecol Sociobiol 62:777-784. doi:10.1007/s00265-007-05032

Johnson BR (2010) Division of labor in honeybees: form, function, and proximate mechanisms. Behav Ecol Sociobiol 64:305-316. doi:10. 1007/s00265-009-0874-7

Johnson BR, Frost E (2012) Individual-level patterns of division of labor in honeybees highlight flexibility in colony-level developmental mechanisms. Behav Ecol Sociobiol 66:923-930. doi:10.1007/ s00265-012-1341-4

Klee J, Besana AM, Genersch E et al (2007) Widespread dispersal of the microsporidian Nosema ceranae, an emergent pathogen of the western honey bee, Apis mellifera. J Invertebr Pathol 96:1-10. doi:10. 1016/j.jip.2007.02.014

Kolmes SA (1984) A quantitative comparison of observational methodologies for studies of worker honeybees. J Apic Res 23:189-198

Lee G, Park JH (2004) Hemolymph sugar homeostasis and starvationinduced hyperactivity affected by genetic manipulations of the adipokinetic hormone-encoding gene in Drosophila melanogaster. Genetics 167:311-323. doi:10.1534/genetics.167.1.311

Martin SJ, Highfield AC, Brettell L et al (2012) Global honey bee viral landscape altered by a parasitic mite. Science 336:1304-1306. doi: 10.1126/science. 1220941
Mayack C, Natsopoulou ME, McMahon DP (2015) Nosema ceranae alters a highly conserved hormonal stress pathway in honeybees. Insect Mol Biol. doi:10.1111/imb.12190

Mayack C, Naug D (2009) Energetic stress in the honeybee Apis mellifera from Nosema ceranae infection. J Invertebr Pathol 100:185-188. doi:10.1016/j.jip.2008.12.001

Mayack C, Naug D (2011) A changing but not an absolute energy budget dictates risk-sensitive behaviour in the honeybee. Anim Behav 82: 595-600. doi:10.1016/j.anbehav.2011.06.022

McDonnell CM, Alaux C, Parrinello H et al (2013) Ecto- and endoparasite induce similar chemical and brain neurogenomic responses in the honey bee (Apis mellifera). BMC Ecol 13:25. doi:10.1186/14726785-13-25

Mersch DP, Crespi A, Keller L (2013) Tracking individuals shows spatial fidelity is a key regulator of ant social organization. Science 340: 1090-1093. doi: $10.1126 /$ science. 1234316

Moore J (2002) Parasites and the behavior of animals. Oxford University Press, New York

Mouritsen KN, Poulin R (2002) Parasitism, community structure and biodiversity in intertidal ecosystems. Parasitology 124:S101-S117. doi:10.1017/S0031182002001476

Mouritsen KN, Poulin R (2010) Parasitism as a determinant of community structure on intertidal flats. Mar Biol 157:201-213. doi:10. 1007/s00227-009-1310-2

Natsopoulou ME, Doublet V, Paxton RJ (2015) European isolates of the Microsporidia Nosema apis and Nosema ceranae have similar virulence in laboratory tests on European worker honey bees. Apidologie. doi:10.1007/s13592-015-0375-9

Naug D (2014) Infected honeybee foragers incur a higher loss in efficiency than in the rate of energetic gain. Biol Lett 10:20140731. doi:10. 1098/rsbl.2014.0731

Nazzi F, Brown SP, Annoscia D et al (2012) Synergistic parasitepathogen interactions mediated by host immunity can drive the collapse of honeybee colonies. PLoS Pathog 8, e1002735. doi:10.1371/ journal.ppat. 1002735

Nelson CM, Ihle KE, Fondrk MK et al (2007) The gene vitellogenin has multiple coordinating effects on social organization. PLoS Biol 5: 0673-0677. doi:10.1371/journal.pbio.0050062

Oster GF, Wilson EO (1978) Caste and ecology in the social insects. Princeton University Press, Princeton

Pohlert T (2015) PMCMR: calculate pairwise multiple comparisons of mean rank sums. R package version 1.1, http://cran.r-project.org/ web/packages/PMCMR/index.html

Poulin R (2006) Evolutionary ecology of parasites. Smithsonian Institution Press, Washington

R Core Team (2014) A language and environment for statistical computing. 3.1.2. edn. R Foundation for Statistical Computing, Vienna, Austria, http://www.R-project.org/

Reber A, Purcell J, Buechel SD et al (2011) The expression and impact of antifungal grooming in ants. J Evol Biol 24:954-964. doi:10.1111/j. 1420-9101.2011.02230.x

Retschnig G, Williams GR, Odemer R et al (2015) Effects, but no interactions, of ubiquitous pesticide and parasite stressors on honey bee (Apis mellifera) lifespan and behaviour in a colony environment. Environ Microbiol. doi:10.1111/1462-2920.12825

Richard F-J, Aubert A, Grozinger CM (2008) Modulation of social interactions by immune stimulation in honey bee, Apis mellifera, workers. BMC Biol 6:50. doi:10.1186/1741-7007-6-50

Richard F-J, Holt HL, Grozinger CM (2012) Effects of immunostimulation on social behavior, chemical communication and genome-wide gene expression in honey bee workers (Apis mellifera). BMC Genomics 13:558. doi:10.1186/1471-2164-13-558

Robinson GE (1992) Regulation of division of labor in insect societies. Annu Rev Entomol 37:637-665. doi:10.1146/annurev.ento.37.1. 637 
Robinson GE (1987) Regulation of honey bee age polyethism by juvenile hormone. Behav Ecol Sociobiol 20:329-338

Rortais A, Tentcheva D, Papachristoforou A et al (2006) Deformed wing virus is not related to honey bees' aggressiveness. Virol J 3:61. doi: 10.1186/1743-422X-3-61

Rueppell O, Bachelier C, Fondrk MK, Page RE (2007) Regulation of life history determines lifespan of worker honey bees (Apis mellifera L.). Exp Gerontol 42:1020-1032. doi:10.1016/j.exger.2007.06.002

Schmid-Hempel P (1998) Parasites in social insects. Princeton University Press, Princeton

Schneider S, Eisenhardt D, Rademacher E (2012) Sublethal effects of oxalic acid on Apis mellifera (Hymenoptera: Apidae): changes in behaviour and longevity. Apidologie 43:218-225. doi:10.1007/ s13592-011-0102-0

Schulz DJ, Huang ZY, Robinson GE (1998) Effects of colony food shortage on behavioral development in honey bees. Behav Ecol Sociobiol 42:295-303. doi:10.1007/s002650050442

Schulz DJ, Sullivan JP, Robinson GE (2002) Juvenile hormone and octopamine in the regulation of division of labor in honey bee colonies. Horm Behav 42:222-231. doi:10.1006/hbeh.2002.1806

Seeley TD (1982) Adaptive significance of the age polyethism schedule in honeybee colonies. Behav Ecol Sociobiol 11:287-293. doi:10. 1007/BF00299306

Shah KS, Evans EC, Pizzorno MC (2009) Localization of deformed wing virus (DWV) in the brains of the honeybee, Apis mellifera Linnaeus. Virol J 6:182. doi:10.1186/1743-422X-6-182

Shen M, Yang X, Cox-Foster D, Cui L (2005) The role of varroa mites in infections of Kashmir bee virus (KBV) and deformed wing virus (DWV) in honey bees. Virology 342:141-149. doi:10.1016/j.virol. 2005.07.012

Siede R, Meixner MD, Büchler R (2012) Comparison of transcriptional changes of immune genes to experimental challenge in the honey bee (Apis mellifera). J Apic Res 51:320-328. doi:10.3896/IBRA.1. 51.4 .05
Smith Trail DR (1980) Behavioral interactions between parasites and hosts: host suicide and the evolution of complex life cycles. Am Nat 116:77-91

Theis FJ, Ugelvig LV, Marr C, Cremer S (2015) Opposing effects of allogrooming on disease transmission in ant societies. Phil Trans R Soc B 370:20140108. doi:10.1098/rstb.2014.0108

Therneau TM, Grambsch PM (2000) Modeling survival data: extending the Cox model. Springer, New York

Therneau TM, Grambsch PM, Pankratz V (2003) Penalized survival models and frailty. J Comput Graph Stat 12:156-175

Tofilski A (2009) Shorter-lived workers start foraging earlier. Insectes Soc 56:359-366. doi:10.1007/s00040-009-0031-3

Tofilski A (2006) Influence of caste polyethism on longevity of workers in social insect colonies. J Theor Biol 238:527-531. doi:10.1016/j. jtbi.2005.06.008

Toth A, Robinson G (2005) Worker nutrition and division of labour in honeybees. Anim Behav 69:427-435. doi:10.1016/j.anbehav.2004. 03.017

Wegener J, Lorenz MW, Bienefeld K (2009) Physiological consequences of prolonged nursing in the honey bee. Insectes Soc 56:85-93. doi: 10.1007/s00040-008-1042-1

Winston M (1987) The biology of the honey bee. Harvard University Press, Cambridge

Woyciechowski M, Kozłowski J (1998) Division of labor by division of risk according to worker life expectancy in the honey bee (Apis mellifera L.). Apidologie 29:191-205. doi:10.1051/apido:19980111

Woyciechowski M, Moroń D (2009) Life expectancy and onset of foraging in the honeybee (Apis mellifera). Insectes Soc 56:193-201. doi: 10.1007/s00040-009-0012-6

Yang Z, Yu Y, Zhang V et al (2015) Octopamine mediates starvationinduced hyperactivity in adult Drosophila. Proc Natl Acad Sci 112:5219-5224. doi:10.1073/pnas.1417838112

Zhukovskaya M, Yanagawa A, Forschler BT (2013) Grooming behavior as a mechanism of insect disease defense. Insects 4:609-630. doi:10. 3390/insects4040609 